\title{
ENHANCED CLASSIFICATION MODEL FOR MULTISPECTRAL OBSERVATIONS FROM THE EARTH
}

\author{
I. E. Villalon-Turrubiates ${ }^{1}$, M. J. Llovera-Torres ${ }^{2}$ \\ ${ }^{1}$ Instituto Tecnológico y de Estudios Superiores de Occidente (ITESO), 45604 Tlaquepaque Jalisco México - villalon@iteso.mx \\ ${ }^{2}$ Universidad Autónoma de San Luis Potosí (UASLP), 78000 San Luis Potosí México - azul.llovera@gmail.com
}

Commission IV, ICWG III/IVb and WG IV/4

KEY WORDS: Identification, Image Classification, Image Processing, Remote Sensing.

\begin{abstract}
:
The image classification procedure to identify remote sensing signatures from a particular geographical region can be performed with an identification model that has the ability to use large datasets to reach an accurate result. This novel methodology is referred to as the Statistical Enhanced Classification algorithm, which has been developed to employ multispectral images based in the statistical supervised learning theory and can be used for applications in environmental monitoring and analysis. This paper presents the performance study of the proposed methodology using both, multispectral synthetic images and multispectral remote sensing images. The obtained results are accurate due to the use of several spectral bands, the use of statistics such as mean and standard deviation for the training classes and for the pixel neighborhood, which provides more robust information, and the decision-making rule that has the ability to decide if the pixel is not belonging to a predefined class, which leads to an accurate decision model.
\end{abstract}

\section{INTRODUCTION}

The applied theory of image processing is a mature and welldeveloped research field of engineering, which provides several developments that has been detailed in many publications. The existing theory offers a manifold of statistical techniques that can be applied for environmental analysis and monitoring; however, in many applications areas there are some unresolved problems related to data processing, especially, when large datasets of information require to be processed in real time (Mather, 2004).

Another problem that needs to be addressed is particularly related to the extraction of the physical characteristics (e.g. land cover, land use, soil, vegetation, water, among others) that are contained within a selected region; afterwards, the analysis of the data and its monitoring can be performed. Moreover, there is a requirement that the model can be robust enough to produce accurate results, and that the methodology can be implemented with a high-performance computing technique in order to reduce the processing time to a suitable value that could be considered as real time.

Real-time is a relative terminology by nature that refers to a level of responsiveness a human may sense as sufficiently immediate, moreover, the concept is completely related to the time in which the processing of the information is needed to be available.

Supervised classification methods (SCMs) are based on external knowledge of the area that is present within an image. The SCMs require some input from the user (or the monitoring administrator) before the chosen methodology can be applied. This input can be obtained from fieldwork, air photo analysis, reports, previous analysis of the region, or from the study of appropriate maps of the area of interest.

The SCMs are implemented using either statistical or neural methodologies (Perry et al., 2002). Statistical algorithms use parameters that are derived from sample data in the form of training classes; these parameters may include: minimum and maximum values of the features, mean and standard deviation of individual clusters, and mean and variance matrices for each class. On the other hand, the neural methods do not rely on statistical information derived from the datasets but are trained on the sample data directly, therefore, these methods make no assumptions of the frequency distribution of the data, requiring more processing time to be trained. Thus, statistical methods are considered to be parametric because they use the statistical parameters derived directly from the training data, and the neural methods are considered to be non-parametric.

\section{STATISTICAL ENHANCED CLASSIFICATION}

The main problem is the situation where the proximity or minimum distance of a pixel to a particular training weight is completely out of certain range, some well-known algorithms will assign the pixel to a class according to their decision rule (Villalon, 2008). However, that range could mean that the pixel belongs to a class that has not been previously considered, which is a recurrent situation in applications of classification techniques to remote sensing images (Fussell et al., 1986).

The problem requires an algorithm with the ability to discern whether a pixel belongs to a defined class, or belongs to an undefined class, improving the classification task through the discarding of irrelevant pixels to the a-priori defined classes.

The solution to this problem is proposed as the Statistical Enhanced Classification (SEC) method, which is based on a statistical supervised classification schema.

The a-priori data for the training process required by the SEC methodology are the number of classes $c$ to be classified, the number of spectral bands $b$ of an image, and the size $m$ of a shifting window that acts as a degree of freedom for accuracy adjustments. 
A means matrix $\mathbf{M}(c \times b)$ and a standard deviation matrix $\mathbf{S}(c \times b)$ are built, which contain, respectively, the mean values $\mu_{c b}$ and the standard deviation values $\sigma_{c b}$ of the classes for each band, where all those values are 8-bit unsigned integers $\left(0 \leq \mu_{c c} \leq 255,0 \leq \sigma_{c c}\right.$ $\leq 255)$ calculated as the mean and standard deviation of a $m \times m$ window that surrounds a candidate pixel of each class and for each band. These matrices $\mathbf{M}$ and $\mathbf{S}$ represents the training weights.

Following the process, the next step is to analyze each pixel within the image for all the spectral bands, using a shifting $m \times m$ window. This window contains the neighborhood that surrounds each pixel of the $n \times n \times b$ image (where $n \times n$ represents the size of the image), determining a group of matrices $\mathbf{M}_{l}, \mathbf{M}_{2}, \ldots \mathbf{M}_{b}$ that defines the means of the neighborhood for each pixel at every spectral band.

Moreover, another groups of matrices $\mathbf{S}_{1}, \mathbf{S}_{2}, \ldots \mathbf{S}_{b}$ that defines the standard deviation of the neighborhood for each pixel at every spectral band are also calculated. These two groups of matrices represent the pixel statistics values.

The main contribution of this proposed methodology is the decision rule employed for the assignment of the classes, which considers that some pixels could belong to an undefined class from the a-priori information.

To compute the output of the classification process, the absolute difference between each value of the pixel statistics matrices and the training weights $\mathbf{M}$ and $\mathbf{S}$ are calculated according to the equation (1).

$$
\operatorname{Diff}_{n n b}=a b s\left(\mathbf{M}_{n n b}-\mathbf{M}_{c b}\right)
$$

Next, for each pixel of the image, the difference values between the pixel and the mean of each of the $c$ training classes are compared between all the $b$ bands, and the minimum value is selected (for each band), this provides the minimum differences between the pixel and each $c$ class $\left(1 \times c\right.$ vector $\left.\mathbf{D}_{c}\right)$.

Also, the mean of those difference values is calculated for each band, and the minimum is selected providing the mean of the differences between the pixel and each $c$ class $\left(1 \times c\right.$ vector $\left.\mathbf{d}_{c}\right)$.

It is supposed that the minimum value of vector $\mathbf{d}_{c}$ defines the class to which the pixel belongs, however, the final step is used to define if the pixel belongs to a different class.

This is performed analysing the values within the $\mathbf{D}_{c}$ vector, if the minimum difference value of the vector is equal to 0 then the pixel belongs to that class $c$, moreover, if the value is different from 0 but is within the threshold defined by the standard deviation matrix for the pixel under study, then the pixel belongs to that class $c$, and finally, if those conditions are not reached then the pixel is not belonging to any of the $c$ classes and will be assigned to a new undefined class (Shkvarko et al., 2007).

This decision rule has the ability to decide whether a pixel maintain statistical homogeneity with the a-priori information (via their means and standard deviations), or it belongs to an undefined class (Jensen, 2005).

The pseudocode that is used for the implementation of the SEC algorithm is presented in the table 1.

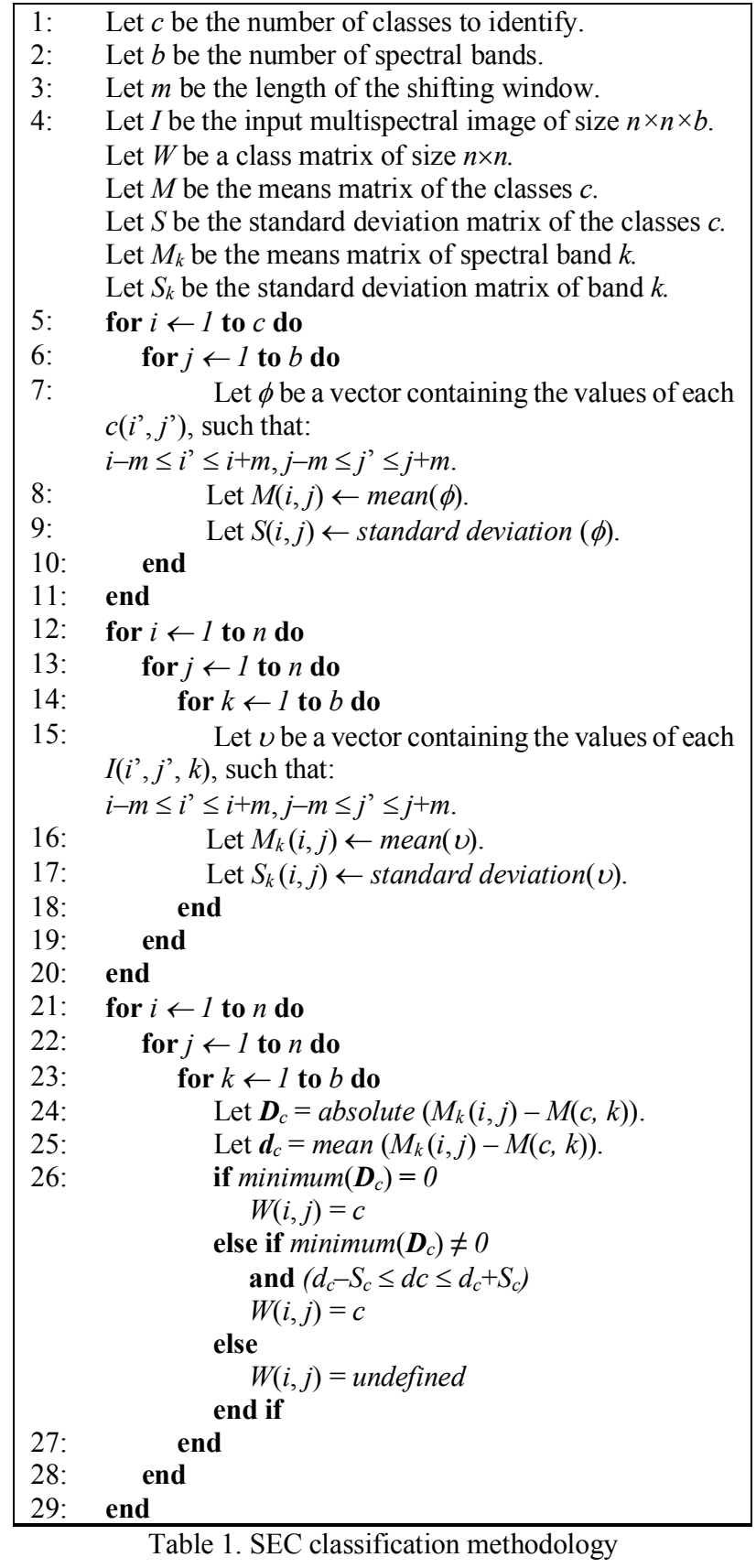

The procedure for the SEC methodology provides the better classification compared with other methods, its strengths are the use of several spectral bands, the use of mean and standard deviation for the training and the pixel neighborhood which provides more robust information, and decision rule that has the ability to decide if the pixel is not belonging to the predefined classes, which leads to an accurate decision model without the use of unsupervised classification techniques.

\section{SIMULATION EXPERIMENT}

In order to analyze the performance of the SEC, a set of four synthetic images are used. The spectral resolution of the images are four spectral bands $(b=4)$, and the image size used for each synthetic image $(n \times n)$ are $400 \times 400$ and $1000 \times 1000$ pixels, this is to compare the computing time required to complete the classification tasks. The file format for the images is TIFF. 
The synthetic image $\boldsymbol{A}$ contains three regions with different patterns (in yellow, blue and dark gray colors) that are used as the a-priori classes $(c=3$ classes $)$. The synthetic images $\boldsymbol{B}, \boldsymbol{C}$ and $\boldsymbol{D}$ includes a small fourth region (light gray color) that is used as an undefined class $(c=3$ classes $)$.

The figure 1 shows the classification results applied to the synthesized images using the SEC method. It is possible to qualitatively verify that the four methodologies perform an adequate classification of the synthetic images, however, some performance differences between the techniques are clear.

The quantitative study is performed by the calculation of the classified percentage obtained with the method and compared with the original quantities of the a-priori classes.

The tables 2 to 5 provides the quantitative results for each synthetic image, respectively. From the results, is possible to confirm that the SEC method is accurate. The methodology was programmed using the Matlab software (MATLAB, 2018) in a personal computer with a $3 \mathrm{GHz}$ Intel Core i7 processor with 16GB of RAM (1,600 MHz, DDR3) and not using parallel processing, therefore, the SEC algorithm can obtain more precise results but requires more computational load and time, which could be a disadvantage for specific applications.

\begin{tabular}{|c|c|cc|}
\hline \multirow{2}{*}{ Synthetic Image $\boldsymbol{A}$} & Original Content & \multicolumn{2}{|c|}{ SEC Method } \\
\cline { 2 - 4 } & $\%$ & $\%$ & Difference \\
\hline Class 1 & 33.61 & 33.76 & 0.16 \\
Class 2 & 24.90 & 24.74 & 0.16 \\
Class 3 & 39.45 & 38.91 & 0.54 \\
Unclassified & 2.05 & 1.59 & 0.45 \\
\hline \multicolumn{2}{|c|}{ Average Percentage Difference $\rightarrow$} & \multicolumn{2}{|c|}{$0.33 \%$} \\
\hline \multicolumn{2}{|c|}{ Total Processing Time $\rightarrow$} & \multicolumn{2}{|c|}{$9.94 \mathrm{sec} .(400 \times 400)$} \\
& $52.00 \mathrm{sec} .(1000 \times 1000)$ \\
\hline
\end{tabular}

Table 2. Comparative table of the class percentages obtained by the SEC method for the synthetic image $\boldsymbol{A}$

\begin{tabular}{|c|c|cc|}
\hline \multirow{2}{*}{ Synthetic Image $\boldsymbol{B}$} & Original Content & \multicolumn{2}{|c|}{ SEC Method } \\
\cline { 2 - 4 } & $\%$ & $\%$ & Difference \\
\hline Class 1 & 33.61 & 33.75 & 0.15 \\
Class 2 & 24.90 & 24.65 & 0.25 \\
Class 3 & 36.69 & 35.99 & 0.70 \\
Unclassified & 4.80 & 4.61 & 0.20 \\
\hline \multicolumn{2}{|c|}{ Average Percentage Difference $\rightarrow$} & \multicolumn{2}{|c|}{$0.32 \%$} \\
\hline \multicolumn{2}{|c|}{ Total Processing Time $\rightarrow$} & \multicolumn{2}{|c|}{$9.97 \mathrm{sec} .(400 \times 400)$} \\
& $49.93 \mathrm{sec} .(1000 \times 1000)$ \\
\hline
\end{tabular}

Table 3. Comparative table of the class percentages obtained by the SEC method for the synthetic image $\boldsymbol{B}$

\begin{tabular}{|c|c|cc|}
\hline \multirow{2}{*}{ Synthetic Image $\boldsymbol{C}$} & Original Content & \multicolumn{2}{|c|}{ SEC Method } \\
\cline { 2 - 4 } & $\%$ & $\%$ & Difference \\
\hline Class 1 & 9.18 & 8.89 & 0.29 \\
Class 2 & 0.16 & 6.98 & 6.82 \\
Class 3 & 79.95 & 79.64 & 0.32 \\
Unclassified & 10.70 & 3.49 & 7.21 \\
\hline \multicolumn{2}{|c|}{ Average Percentage Difference $\rightarrow$} & \multicolumn{2}{|c|}{$3.66 \%$} \\
\hline \multicolumn{2}{|c|}{ Total Processing Time $\rightarrow$} & \multicolumn{2}{|c|}{10.39 sec. $(400 \times 400)$} \\
& 58.34 sec. $(1000 \times 1000)$ \\
\hline
\end{tabular}

Table 4. Comparative table of the class percentages obtained by the SEC method for the synthetic image $C$

\begin{tabular}{|c|c|cc|}
\hline \multirow{2}{*}{ Synthetic Image $\boldsymbol{D}$} & Original Content & \multicolumn{2}{|c|}{ SEC Method } \\
\cline { 2 - 4 } & $\%$ & $\%$ & Difference \\
\hline Class 1 & 31.11 & 30.72 & 0.39 \\
Class 2 & 30.69 & 30.55 & 0.14 \\
Class 3 & 31.01 & 30.74 & 0.27 \\
Unclassified & 7.19 & 6.99 & 0.19 \\
\hline \multicolumn{2}{|c|}{ Average Percentage Difference $\rightarrow$} & \multicolumn{2}{|c|}{$0.25 \%$} \\
\hline \multicolumn{2}{|c|}{ Total Processing Time $\rightarrow$} & $10.14 \mathrm{sec} .(400 \times 400)$ \\
& $50.18 \mathrm{sec} .(1000 \times 1000)$ \\
\hline
\end{tabular}

Table 5. Comparative table of the class percentages obtained by the SEC method for the synthetic image $\boldsymbol{D}$

\section{GEOSIMULATION}

To probe the efficiency of the proposed methodology, a real multispectral remote sensing (MRS) image is processed. The scene was provided by the SPOT-5 satellite through its Mexican office SEMAR (from its Spanish acronym "Secretaría de Marina") under the ERMEXS program (from its spanish acronym "Estación de Recepción México de la Constelación SPOT") (ERMEXS, 2018).

SPOT Imagery (from its french acronym "Système Pour L'Observation de la Terre") is the worldwide distributor of geographic information products and services derived from the SPOT Earth observation satellites (SPOT, 2018).

The image corresponds to the geographical region of the Guadalajara Metropolitan area (Mexico). The spatial resolution of the image is 20 meters (spectral mode Hi) for a $6000 \times 6000$ pixels image, and the spectral resolution of 4 spectral bands corresponds to:

Band XS3: Near infrared (multispectral mode), wavelengths from 0.79 to $0.89 \mu \mathrm{m}$.

Band XS2: Red (multispectral mode), wavelengths from 0.61 to $0.68 \mu \mathrm{m}$.

Band XS1: Green (multispectral mode), wavelengths from 0.50 to $0.59 \mu \mathrm{m}$.

Band SWIR: Short-wave infrared (multispectral mode), wavelengths from 1.58 to $1.75 \mu \mathrm{m}$.

The figure 2 shows the original MRS image in false colour, and figure 3 shows the classification result applied to the MRS image using the SEC method.

Once again, in a qualitatively analysis from the results, it is possible to verify that the methodology performs an adequate classification of the MRS image and has been able to achieve accurate results. For the classification process, three classes are selected and are:

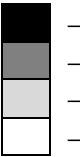

- Water bodies within the MRS scene.

- Mountainous terrain within the MRS scene.

- Urban zone within the MRS scene.

- Urban zone within the MRS scene.

The table 6 shows the processing time for the SEC method with different sizes of the MRS image (in seconds), and was implemented with the same parameters as the synthetic images. 


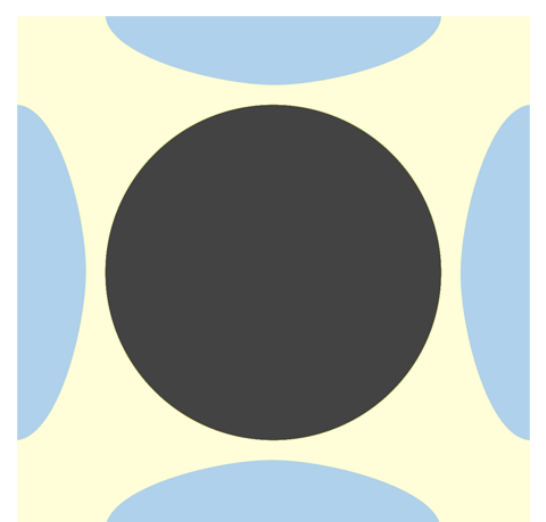

Synthetic image $\boldsymbol{A}$.

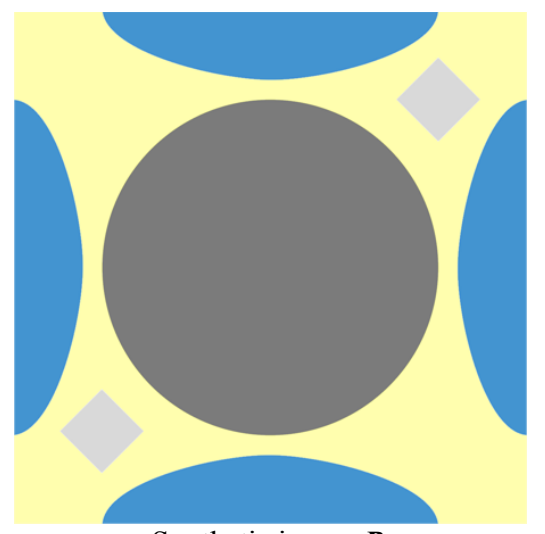

Synthetic image $\boldsymbol{B}$.

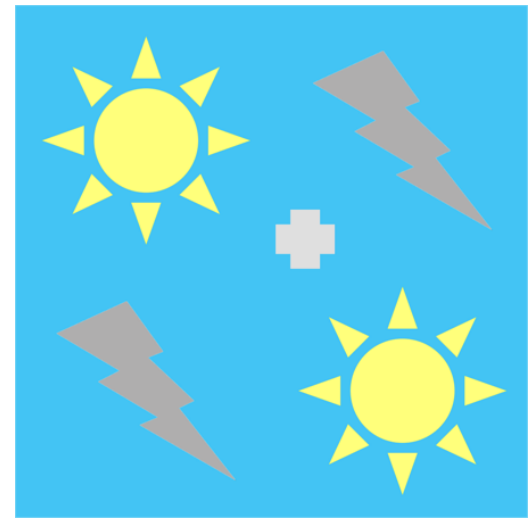

Synthetic image $\boldsymbol{C}$.

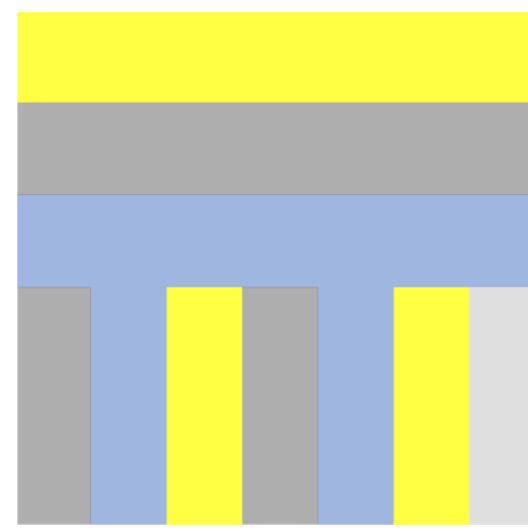

Synthetic image $\boldsymbol{D}$.

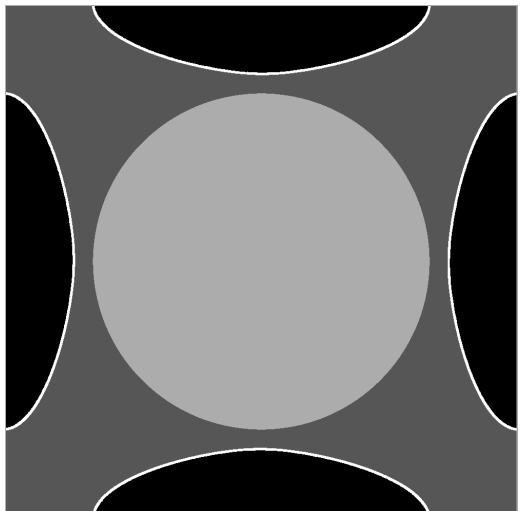

Image $\boldsymbol{A}$, SEC Method.

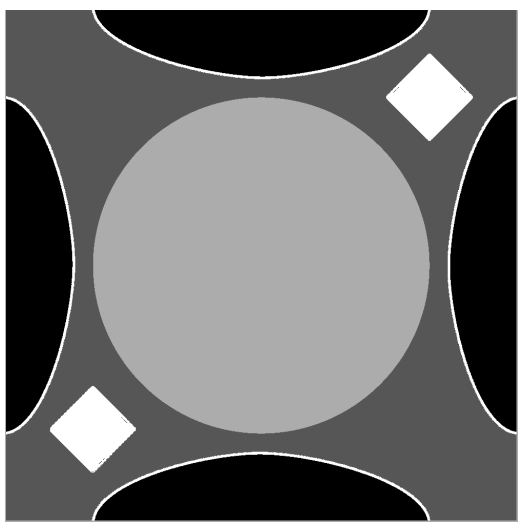

Image $\boldsymbol{B}$, SEC Method.

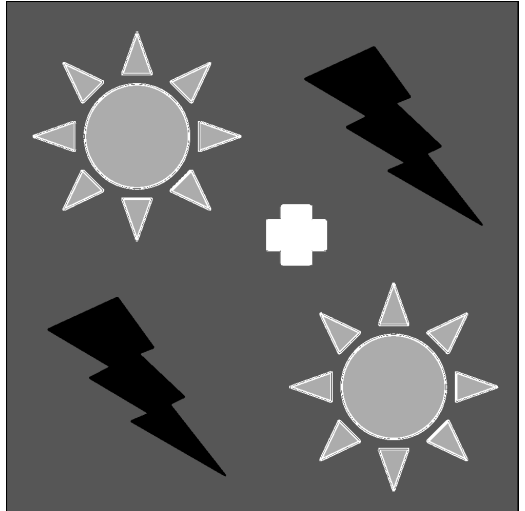

Image $\boldsymbol{C}$, SEC Method.
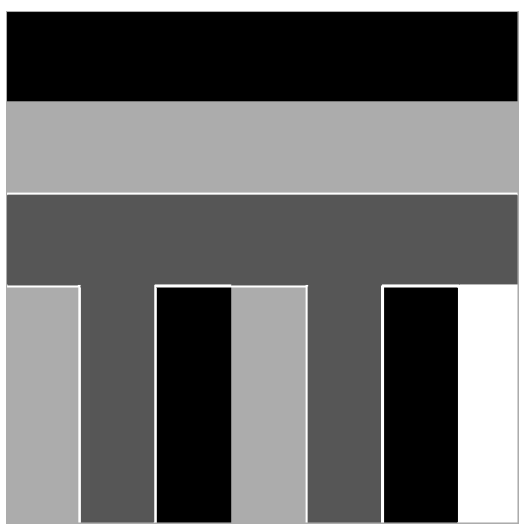

Image $\boldsymbol{D}$, SEC Method.

Figure 1. Synthetic images $\boldsymbol{A}, \boldsymbol{B}, \boldsymbol{C}$ and $\boldsymbol{D}$ (presented in false colour) and the classification results using the SEC methodology. 


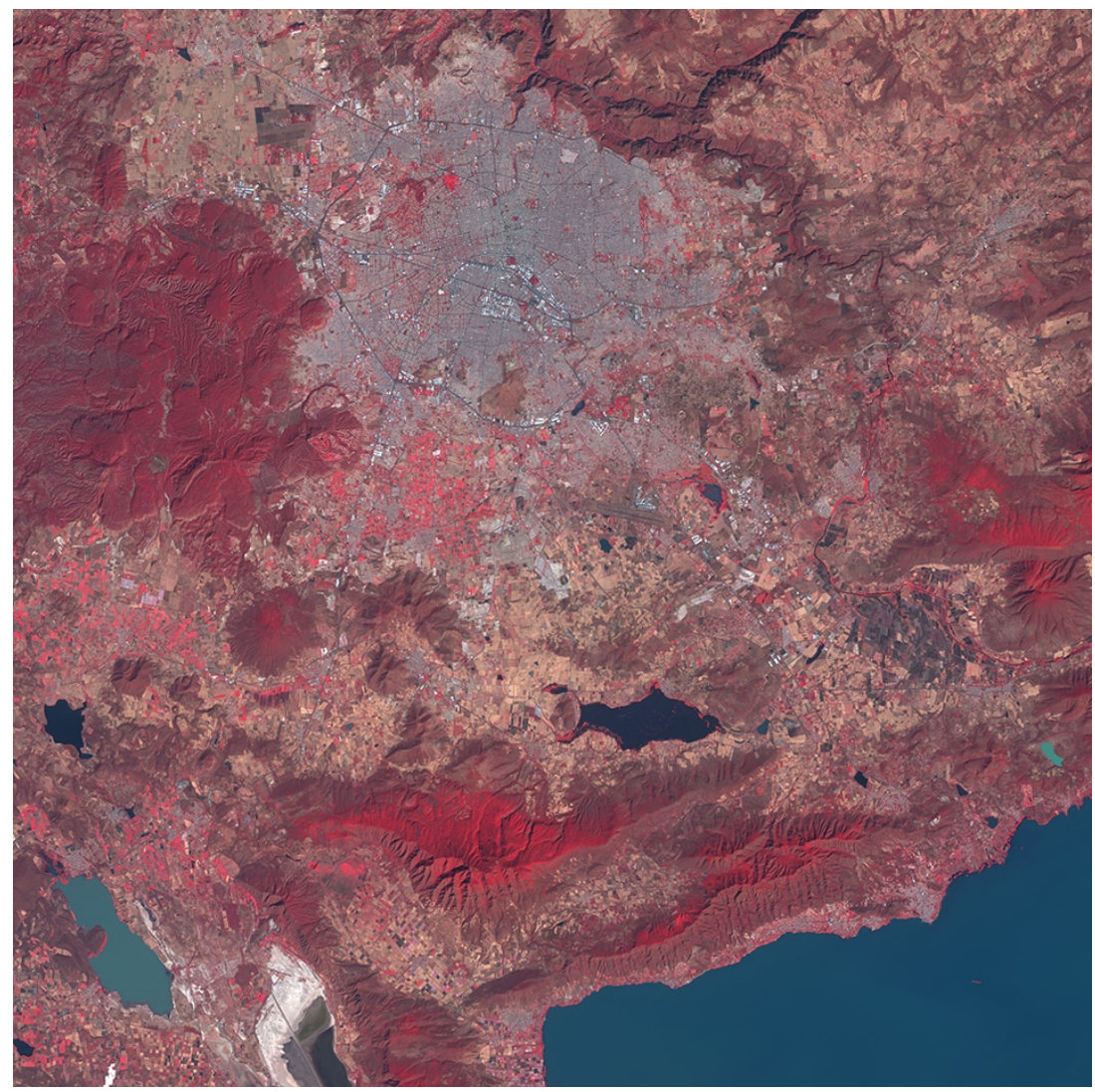

Figure 2. MRS image (presented in false color).

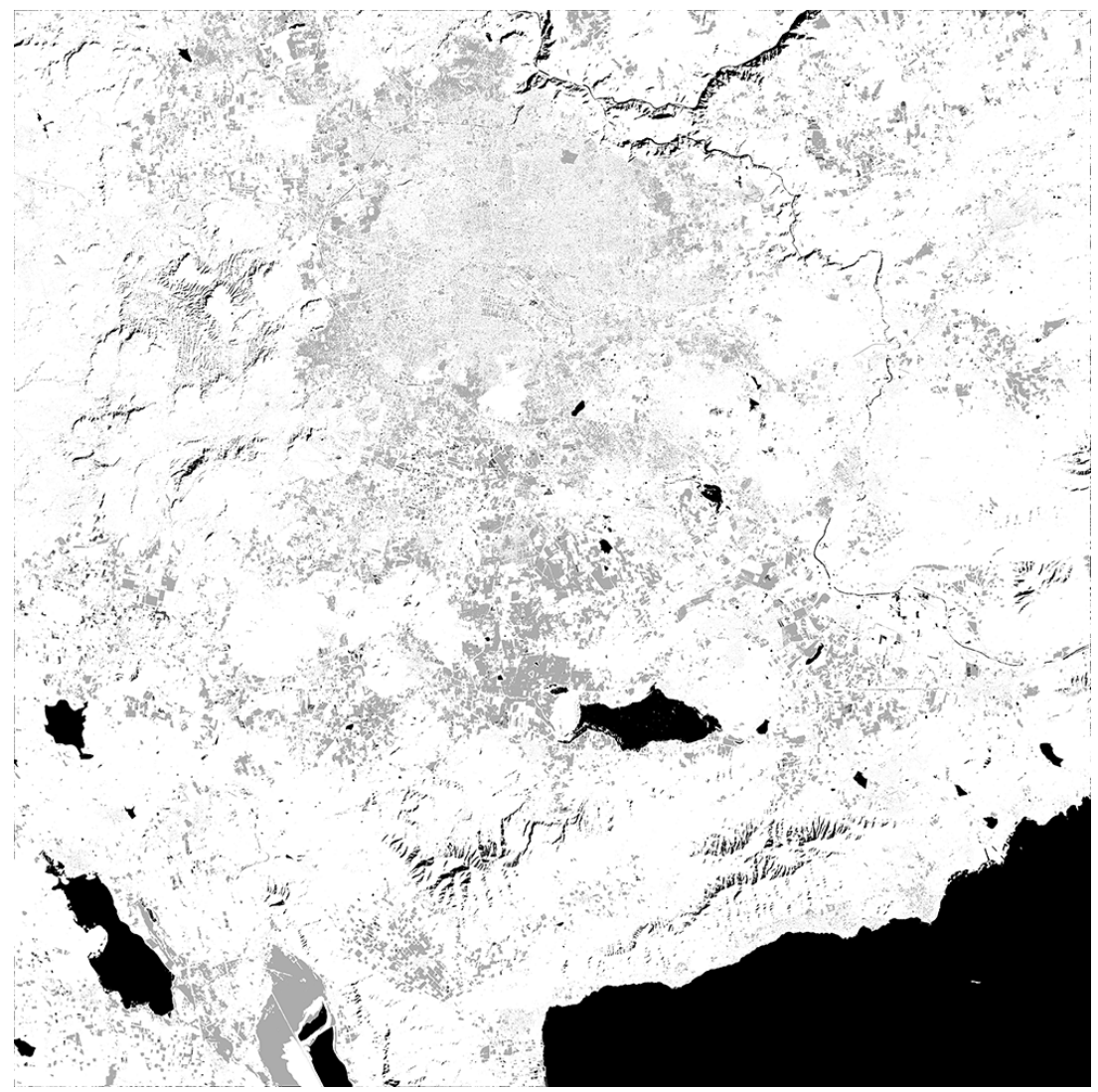

Figure 3. Classification results of the MRS image using the SEC methodology. 


\begin{tabular}{|c|c|}
\hline Image Size & SEC \\
\hline $400 \times 400$ & 10.63 \\
$1000 \times 1000$ & 47.64 \\
$6000 \times 6000$ & $1,523.63$ \\
\hline
\end{tabular}

Table 6. Comparative table of the classification time applied to the MRS scene (the results are expressed in seconds)

\section{CONCLUDING REMARKS}

From the simulation results applied to the synthetic images, it is possible to verify the classification capabilities of the proposed SEC methodology, showing that, qualitatively, the results are accurate due to the use of several spectral bands, the use of mean and standard deviation for the training and the pixel neighbourhood, which provides more robust information, and the decision rule that has the ability to decide if the pixel is not belonging to the predefined classes, which leads to an accurate decision model without the use of unsupervised classification techniques.

The reported results are some representative results from a large validation process using several synthetic images. The application to a real MRS image probed qualitatively that the classification with the SEC method remains as the more accurate.

However, a more intense validation process is required in order to define the most robust size of the shifting window $m$ that acts as a degree of freedom for accuracy adjustments.

Also, the validation with on-site a-priori information to quantitatively analyse its accuracy is also needed. Even when the proposed SEC method provides an accurate classification, the main problem is the processing time needed to reach the results.

Applied to a real MRS image, the process required around 25 minutes (for a $6000 \times 6000$ pixels image) to produce the results using an average computer, this could not be suitable for certain applications where a reduced time response is vital.

\section{FUTURE WORK}

The results reported on this paper shows the qualitative and quantitative analysis of the overall performance of the SEC method applied to remote sensing data for MRS images obtained from the Earth. The application as an auxiliary tool in geophysical information retrieval and data interpretation for land use management and analysis, and a more extensive quantitative analysis of the results, and a comparison with different methodologies from the state of the art are a matter of further studies.

\section{ACKNOWLEDGEMENTS}

The author would like to thank the Instituto Tecnológico y de Estudios Superiores de Occidente (ITESO) of Mexico for the resources provided for this research under the project titled "Desarrollo de modelos adaptivos para el procesamiento digital de señales multiespectrales de percepción remota y su implementación como software de alto desempeño".

\section{REFERENCES}

ERMEXS 2018. Estación de Recepción México de la Constelación SPOT - Secretaría de Marina SEMAR / Armada de México, http://www.ermexs.siap.gob.mx (1 January 2018).

Fussell, J., Rundquist, D. and Harrington, J., 1986. On defining remote sensing. Journal of Photogrammetric Engineering \& Remote Sensing, Vol. 52, 1507-1511.

Jensen, J., 2005. Introductory Digital Image Processing: A Remote Sensing Perspective, Prentice-Hall, U.S.A.

Mather, P., 2004. Computer Processing of Remotely-Sensed Images. U.S.A.: John Wiley \& Sons.

MATLAB Mathworks, 2018. Mathematical Analysis Software, Version 2008a, https://www.mathworks.com (1 January 2018).

Perry, S., Wong, H. and Guan L., 2002. Adaptive Image Processing: A Computational Intelligence Perspective. U.S.A.: CRC Press.

Shkvarko, Y., Villalon-Turrubiates, I., 2007. Remote Sensing Imagery and Signature Fields Reconstruction via Aggregation of Robust Regularization with Neural Computing. In Lecture Notes in Computer Sciences, Blanc-Talon J., Philips W., Popescu D., Scheunders P., Springer-Verlag, pp. 235-246.

SPOT Satellite Imagery and Smart Mapping Solutions, 2018. http://www.spotimage.com (1 January 2018).

Villalon-Turrubiates, I., 2008. "Weighted Pixel Statistics for Multispectral Image Classification of Remote Sensing Signatures: Performance Study", Proceedings of the $5^{\text {rd }}$ IEEE International Conference on Electrical Engineering, Computing Science and Automatic Control (CCE), Mexico City, pp. 534539. 